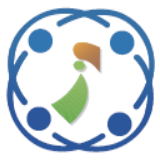

\title{
A Case Study on Thailand's New Teleprotection Regulation: Direct Transfer Trip Application in Distributed Generation Network
}

\author{
Naradon Chotiwanaporn ${ }^{1} \quad$ Chow Chompoo-Inwai ${ }^{1 *}$ \\ ${ }^{1}$ Electrical Engineering Department, Faculty of Engineering, \\ King Mongkut's Institute of Technology Ladkrabang, Bangkok, 10520, Thailand \\ * Corresponding author’s Email: chompooc@gmail.com
}

\begin{abstract}
This research paper presents the new idea of applying the transmission line teleprotection scheme called direct transfer trip (DTT) technique to use with a closed-loop distribution network. A large-scale 163MW gas turbine power plant connected to a three-substation closed-loop distribution network was chosen to be a case study here. A newly-proposed DTT configuration, DTT logic diagram and circuit breaker trip matrix had been redesigned and presented in this paper. For the proposed DTT configuration, several remote terminal units will be installed at every substation connected to the distribution network, whereas the logic controller will be installed at the selected power plant. A huge advantage over the conventionally-installed zoning peer-to-peer protection scheme is that the proposed DTT logic controller is able to almost instantaneously compute and send out the clearing faults command, regardless of fault locations in this particular closed-loop distribution network, while the conventional one cannot in many cases. Additional benefit is that the proposed DTT teleprotection scheme completely prevent the generators from operation in island mode resulting in minimal lost and damages caused from this circumstance. The relay test tool is used to verify the proposed DTT protective capabilities and speed. The simulation and commissioning test results confirm the proper functionality of the proposed technique. The implementation to the real field has been done and presented here as well. What we have learned from this case study shall be a very good guideline for other similar circumstances.
\end{abstract}

Keywords: Direct transfer trip, Teleprotection, Island mode, IEC-60834, IEEE-1547, IEEE-C37.236.

\section{Introduction}

\subsection{Electric utilities in Thailand}

In Thailand, three state utilities are responsible for the overall country's electrical power system namely EGAT (the Electricity generating authority of Thailand), MEA (the Metropolitan electricity), and PEA (the Provincial electricity authority). EGAT is mainly responsible for generations and high voltage transmission line systems. MEA is responsible for the metropolitan area's distribution and medium to low voltage transmission system. And PEA is responsible for the rest of the country's distribution and medium to low transmission line systems.
According to Thailand Power Development Plan (PDP), governed by the Ministry of Energy, Thai government launched the new policy to enhance Thailand's electrical power system stability and security by purchasing more electrical power from local private sectors i.e., Independent power producers (IPP), Small power producers (SPP) and Very small power producers (VSPP) [1]. EGAT is responsible for purchasing electrical power from IPPs and SPPs, while PEA is responsible for purchasing from IPPs, SPPs and VSPPs, whereas MEA is responsible for purchasing from only VSPPs. Each utility has its own policy and regulations in electricity purchasing. All of the power purchase agreement (PPA) owners have an option to connect and sell electricity to only one utility. IPPs or SPPs owner can choose between 
EGAT and PEA. SPPs and VSPPs owner is able to choose between PEA and MEA. IPPs/SPPs/VSPPs must update its systems to comply with connected grid regulations. Since a selected power plant studied in this paper is in the scale of SPP and is located in the eastern region of Thailand; therefore, it must follow the rules of thumbs in the PEA territory.

As for PEA new regulations for interconnection launched in late of the year 2015, there are two main protection schemes required for PEA contractors (IPPs/SPPs). One is to have at least the simplest teleprotection capability that can prevent the DGs from running in island mode within 0.1 second. And another is the ability to link up with PEA supervisory control and data acquisition system (PEA SCADA). Both of the teleprotection and SCADA communications must be done via PEA fibre-optic cable network.

\subsection{Teleprotection schemes}

The idea of teleprotection scheme in general power system was originally proposed in IEEE transaction since year 1992 by D. Fischer [2]. The main idea at that time was to apply the principle telecommunication concept to the traditional protective relaying system by using the prototype of digital teleprotection unit called DTU. The formerly proposed DTU was used in the point-to-point or peer-to-peer protection scheme via the microwave technique which was the best scene at the time. During mid to late 1990, IEC group of committees released the standard about the teleprotectionrelevant equipment and test procedures [3-4].

Schweitzer described the idea and concept of using teleprotection schemes in transmission line networks, sometimes called line protection scheme. Teleprotection concept has been mostly applied in general power system transmission line network for many years to enhance protection system's speed, reliability and sensitivity. The following example teleprotection methods have been applied in transmission line networks [5-6]:

- Permissive overreaching transfer trip (POTT). In this scheme, an overreaching element keys a permissive transfer trip. A trip signal is issued for operation of an overreaching element and reception of the permissive transfer trip.

- Permissive underreaching transfer trip (PUTT). In this scheme, an underreaching element keys a permissive transfer trip. A trip signal is issued for operation of an underreaching element and reception of the permissive transfer trip.
- Directional comparison blocking (DCB). In this scheme, a reverse-looking element keys a block. A trip is issued for operation of an overreaching element without reception of a block.

- Directional comparison unblocking (DCUB). In this scheme, a block is keyed during normal operation. An unblocked status is keyed when the overreaching element operates. A trip is issued for operation of the overreaching element and reception of unblocked status. In addition, the overreaching element is allowed to trip if neither the block nor unblock is received for a finite period. DCUB is therefore a form of POTT that allows a trip for a simultaneous occurrence of a forward fault and a loss of communication.

- Direct underreaching transfer trip (DUTT). In this scheme, an underreaching element sends a direct transfer trip command. A trip is issued upon reception of the transfer trip signal.

- Direct transfer trip (DTT). In this scheme, remote breakers are tripped upon reception of the trip command. Breaker failure protection is a typical example.

All of these schemes require a communication channel. We refer to a channel as the physical medium used to convey any signal needed in the teleprotection schemes. The most commonly-used for mediums are power line carrier, leased phone lines, microwave radio and fiber-optic cable. The choice of these channels would normally be selected based on availability, speed, cost and security.

In 2006, Jager and et al proposed adaptive technique to detect power system faults using distance relay and claimed that their technique is able to detect faults faster than the conventional POTT and PUTT techniques. By changing the socalled solid state relays to the more modern digital relays with the basic teleprotection schemes [7]. In 2009, Andre Luiz P.de Oliveira proposed the use of Siemens device called real time digital simulator to simulate how digital distance relay works to expand the knowledge of how the teleprotection scheme works [8]. In 2011, M. Condadad and et al proposed the guideline for selecting best distance relay teleprotection schemes among POTT, PUTT, DCB and DUTT including how to compute the proper relay tripping time in IEEE conference [9]. In 2012, Schweitzer and et al proposed the application of using $915 \mathrm{MHz}$ radio communication in distributed generation teleprotection schemes which mainly mentioned the simulation results of using radio wave as mediums on six different teleprotection schemes: POTT, PUTT, DCB, DCUB, DUTT and DTT to prevent the damage in distributed generators 
when the short circuit fault occurred at the point of main power supply [10]. In 2013, Antonova and et al proposed the review of using communication technique with relay protection schemes in five different aspects in order to see the effects of variety communication systems by different types of faults [11]. In 2014, S. Roesler and R. Lobo proposed the viability on line current differential over packing switched networks. They explained how to do the differential relay commissioning test using the GPS time between two substations [12]. In 2015, C. Chompoo-inwai and et al proposed the use of DTT teleprotection scheme with Mirrored Bit protocol in an opened-loop distribution network between two substations [13].

\subsection{Challenges}

The current status in Thailand is that most of the longstanding PEA's distribution networks utilize the conventional extension zone protection scheme. After PEA announcement of the new regulation for teleprotection capability, there are some attempts trying to apply both PUTT and POTT concepts to meet PEA's new requirement which is okay when there is no DGs connected to such a distribution network. The drawback of those aforementioned protection schemes is that when faults occurred outside the zone protection, the circuit breaker at some points in the network will either trip open too slow or no trip at all. During those faults occurring time, if there were some DGs connected to such a distribution network, it is a very high possibility that the generators will be running in island mode which normally causes a big lost and damages.

As mentioned before, the DTT teleprotection concept is mostly used in the line protection system. Some research papers applied the DTT application in the distribution networks but all of them use simple opened-loop with only two nodes/buses configuration. In this research paper, we proposed the use of modified DTT teleprotection scheme to a closed-loop distribution network with DGs and multi substations. The main goals are to prevent and minimize damages from DGs' generators running in island mode and also to enhance the distribution system protection speed, stabiltiy and security. The key concept of the proposed DTT is to gather the circuit breaker status at every bus in the network and then pass trough the newly-designed logic processor installed at the DGs site. The logic processor will then compute the desired output signals to send out trip command to each of circuit breaker in the substation. All of the communications links will be done via PEA's fiber-optic cable system. All of the
DTT teleprotection configuration and DTT logic diagram must be redesigned to insure the stability regardless of fault locations at any node in the closed-loop network. Not only similarly smart features of the DTT capabilities must be achieved, but also the speed and accuracy of the proposed DTT must be superior to the conventional one. We also proposed the methodology of how to do the commissioning process and test results to verify the speed, capability and stability of the newly-designed teleprotection concept. A variety of fault locations simulation and commissiong test results presented later in this paper show a very promising potential.

\section{Existing distribution network at LPP}

Lamchabang power plant (LPP) is connected to PEA $115 \mathrm{kV}$ closed-loop substations called PEAAPB-LCA-LCB loop. The power system network diagram of LPP is illustrated in Fig. 1. The direct connection between LPP and PEA loop is at LCA. LPP originally has only one main unit of generation (LPP1) with the capacity of 113 MW. LPP now increases its power production capacity to $163 \mathrm{MW}$ (additional $50 \mathrm{MW}$ under the name LPP2 since 2013). Both of the generation units: LPP1 and LPP2 will be combined and recalled as LPP. Overall generating system now consists of two main units and six generators (G1-G6) with a total capacity of 163 MW.

\subsection{Existing protection scheme at LPP}

The conventional zoning protection scheme at LPP before expanding power production relies only on three simple protective relays i.e., distance relay (21), directional relay (67) and differential relay (87)

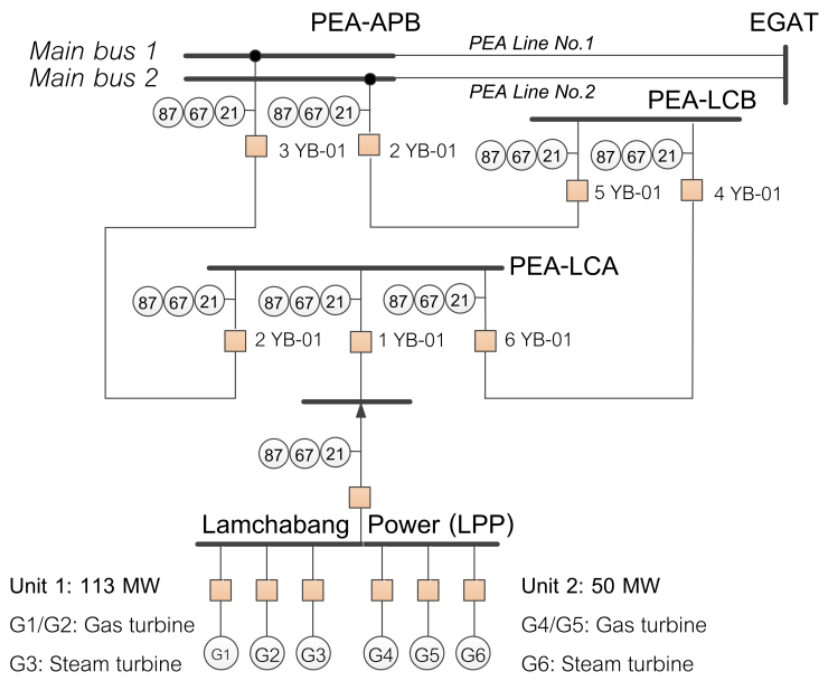

Figure. 1 A closed-loop PEA-LPP distribution network 


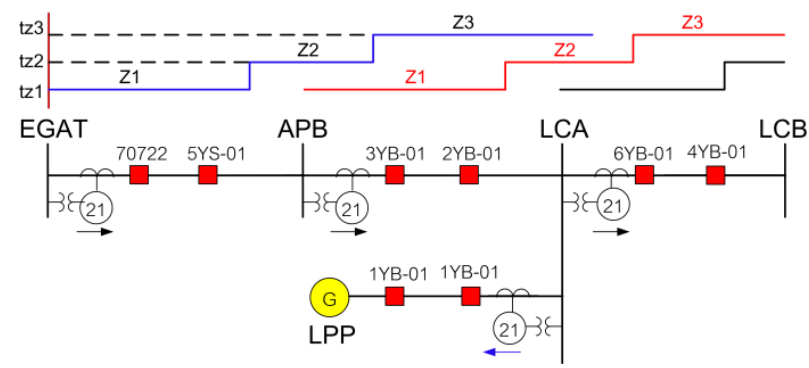

Figure. 2 The conventional zone protective scheme at LPP before expanding power production capacity

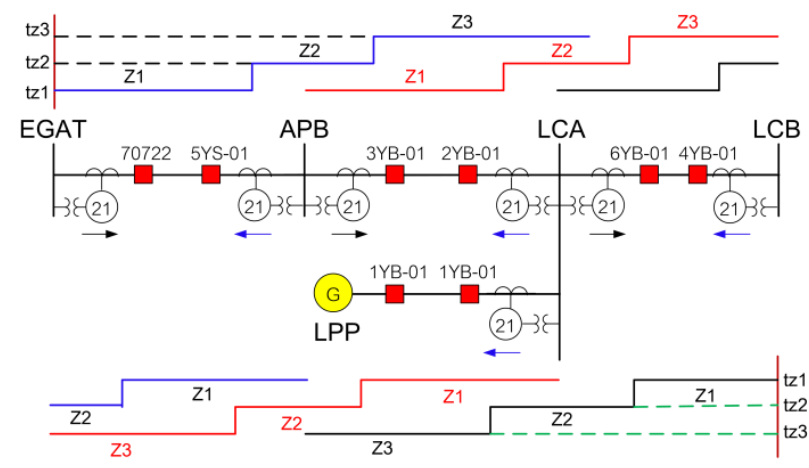

Figure. 3 The improved peer-to-peer protection scheme

without any teleprotection, this simple protective scheme can be seen in Fig. 2.

The drawback of such a simple protection scheme shown in Fig. 2 is that, often, when faults or disturbances occurred outside the protection zone 1 , the protective relay at LPP is not working, resulting in generators at LPP operating in island mode. This results in generators minimal to severe damages.

After the installation of G4-G6 to increase the power production to $163 \mathrm{MW}$, the improved version of the conventional zoning protection scheme had been installed at LPP. A peer-to-peer protection scheme as shown in Fig. 3 with two directional distance relays was installed at the time. The hypothesis is that a peer-to-peer protection scheme will help improving the protection speed, reliability and performance; and more importantly, to prevent the generators at LPP from operating in island mode which will result in lower maintenance cost. About a year after installation of the peer-to-peer protection scheme, LPP engineering team found out that the issue of generators operating in island mode remains. The already installed peer-to-peer protection scheme is still not a solution.

\subsection{Detail on connection in all substations at PEA-LPP closed-loop network}

Fig. 4 presents the detailed connection in PEAAPB substation. At APB, there is only one incoming line from EGAT via PEA $115 \mathrm{kV}$ overhead redundant lines with the choices of selected either PEA Bus No.1 or PEA Bus No.2. There are four outgoings at this substation: Outgoing No.1 (to load via 1YB-01, normally close), No.2 (to LPB via 2YB-01 which normally open as spare), No.3 (to LPA via 3YB-01, normally close) and No.4 (to load via 4YB-01, normally close), respectively. It should be noted here that, the red line colour in this paper represents the GCB or LINE status as normally close and the black line represents vice versa.

Fig. 5 shows the detailed connection in PEALCA substation. At LCA, there are two $115 \mathrm{kV}$ incoming lines from PEA-APB (via 2YB-01, normally close) and IPP-LCP (via 1YB-01, normally close). There are four outgoings: No.1 (to load via 4YB-01, normally close), No.2 (to LPB via 6YB-01, normally close), No.3 (to TP1 via 3YB-01, normally close) and No.4 (to TP2 via 5YB-01, normally close), respectively. The reserved and transfer GCB BYB-01 shown here will be in operation for the maintenance purpose only.

Fig. 6 illustrates the detailed connection in PEALCB substation. At LCB, there are two incomings: one from LCA (via 4YB-01, normally close) and another from APB (via 5YB-01, normally open). There are three outgoings: No.1 (to TP1 via 1YB-01, normally close), No.2 (to TP2 via 3YB-01, normally close), and No.3 (to PATTAYA via 6YB-01, normally close), respectively.

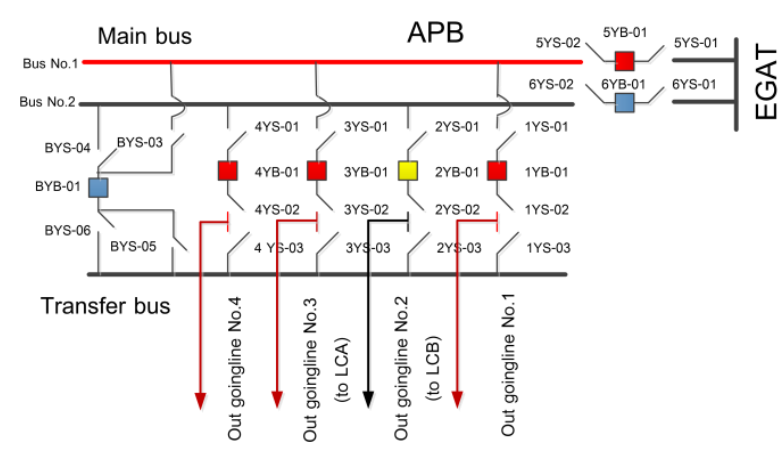

Figure.4 Network diagram of PEA-APB substation

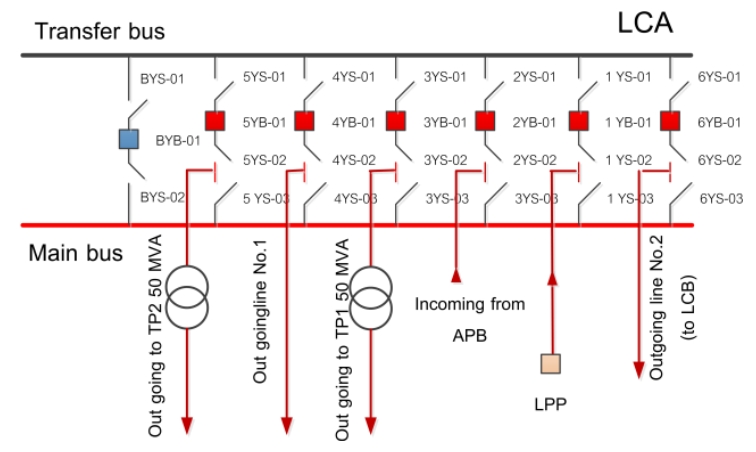

Figure.5 Network diagram of PEA-LCA substation 


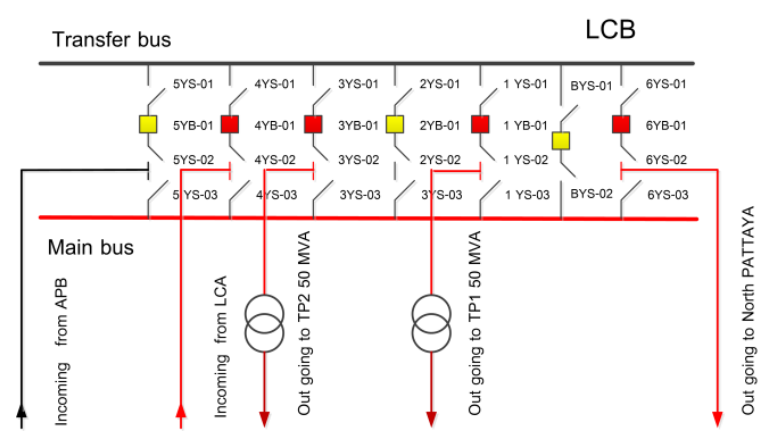

Figure.6 Network diagram of PEA-LCB substation

\section{Proposed DTT teleprotection scheme}

Although we proposed the same name DTT, but unlike the DTT concept in the protective scheme in transmission line networks which usually installed between two substations right next to each other. What we proposed here is to install the remote terminal units at every connected bus in this particular distribution closed-loop network which results in complete coordination of the distribution relays regardless of fault locations at any zone protection. The communication medium in this particular case is done via PEA's fiber-optic network.

\subsection{Proposed DTT configuration}

To implement DTT technique to this particular scenario, the Remote terminal units must be installed at every PEA substation connected to this loop. All of the available statuses of protection devices at each substation i.e., the gas circuit breaker (GCB) and the disconnecting switch (DS) will be sending out to the logic processor installed at the LPP power plant. The logic processor will then determine what to do with all the LPP circuit breakers and protection devices based on the status acquiring from other protection devices from other substations in this particular loop. More details on how the logic processor works will be explained in section 3.2. The main goal of this DTT concept is to protect the LPP's generators from operating in Islanding mode when there are some faults occurred in any other location for this power system network; especially, when the sources of power from PEA is disconnected from the system. Another positive point is that when implementing this DTT concept to the system, we expect faster operating time in any protection scenario.

Fig. 7 illustrates the schematic diagram of the proposed DTT technique with Mirrored bits® protocol protection scheme. In this paper, we apply the SEL-2505/SEL-2506 as remote terminal units at

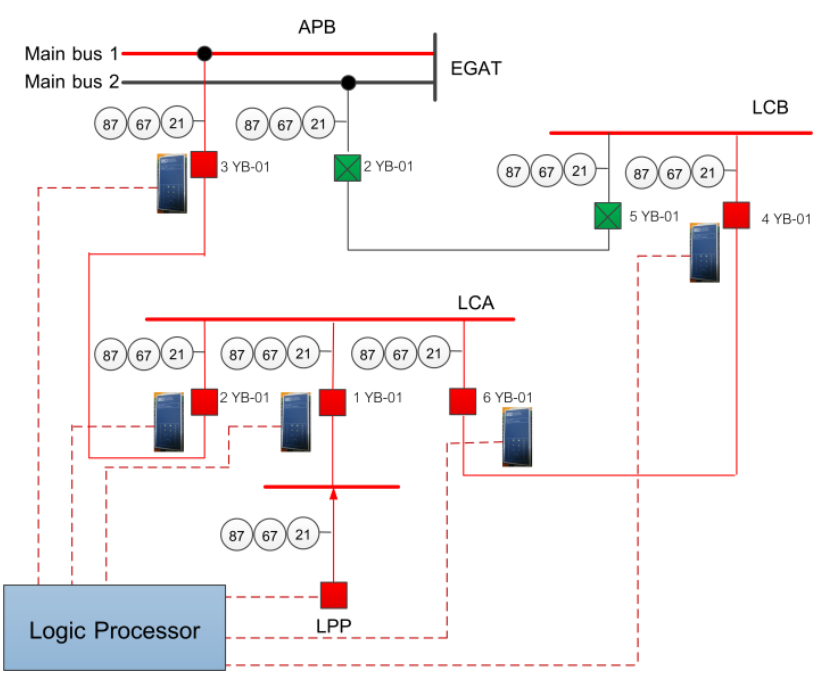

Figure.7 The proposed single line diagram of a DTT technique with mirror bit protocol protection scheme

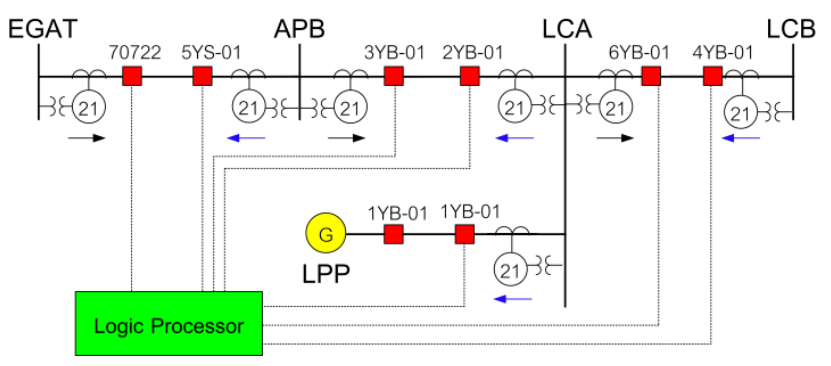

Figure. 8 The simplified single line diagram of the proposed DTT configuration

each connected substation and the SEL-2100 as a logic processor at LPP station. All of the telecommunication cables were connected via PEA fiber-optic network. Fig. 8 presents the simplified version of the proposed DTT teleprotection scheme as described in Fig. 7.

\subsection{Proposed DTT logic diagram}

The logic diagram of the proposed DTT configuration for this particular case in LPP power plant is illustrated in Fig. 9. The logic diagram for this system must be redesign rather than the conventional DTT one.

Logic diagram shown in Fig. 9 was designed based on the concept of the DTT technique which has main purpose to protect the Generators of LPP power plant from operating in Island mode when any kind of fault occurred in PEA closed-loop network. The second goal is to assure the speed of GCB tripping time to be faster than the conventional peer-to-peer protection scheme to also protect the Generators of LP power plant from damages in Islanding mode. Presented logic diagram here 


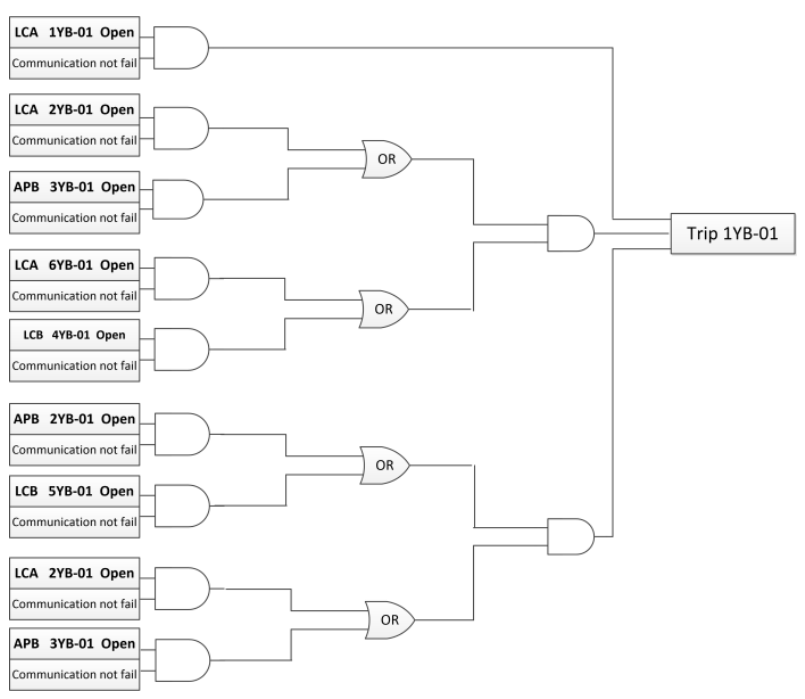

Figure.9 Logic diagram for DTT configuration

covers mostly the main and critical criteria for trip signal to open the GCB 1YB-01 (at LCA).

For example, the GCB 1YB-01 must trip instantaneously while DTT is in place when fault occurred at the transmission line level or PEA incoming Bus. Another example is that if there is a fault occurred at 2YB-01 (LCA) or 3YB-01 (APB), the DTT protection scheme must recheck to see whether or not the GCB 6YB-01(LCA) or 4YB01 (LCB) is opened. If the above condition is true, The DTT logic controller must send out the trip signal to open the GCB 1YB-01 simultaneously.

\subsection{Proposed DTT circuit breaker details}

Fig. 10, Fig. 11 and Fig. 12 illustrate the single line diagram including the incoming and outgoing details of each substation connected to this PEA loop (APB, LCA, LCB, respectively).

Fig. 10(a) represents the modified single line diagram of APB substation to meet up with the specification of the proposed DTT technique. Fig. 10(b) also illustrates the logic algorithm of the trip matrix and trip circuit at APB substation.

This modification is done by redoing the logic function in according to DTT technique criteria using the acquired signals from GCB (Gas circuit breaker) and DS (Disconnecting switch) auxiliary contacts. It is then sending out the trip signal to open/close the GCB 1YB-01 (to LCA).

At OUT1 and OUT2 terminals, the trip signals will be sending out to open GCB 1YB-01 (LCA) when DS 2YS-03 (LCA) or GCB 2YB-01 (APB) opened. Another case to send out the trip signals is when the transfer bus function is in use meaning that the GCB BYB-01 or GCB 6YB-01 are opened, see also Fig. 10(b).

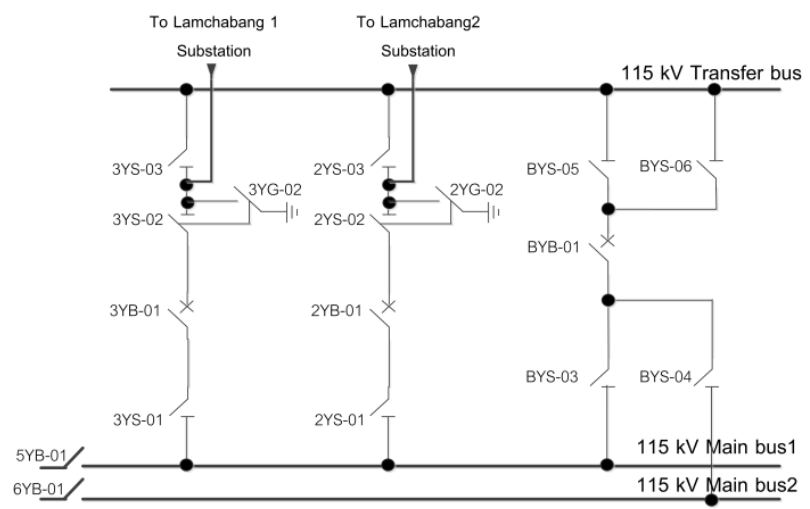

(a)
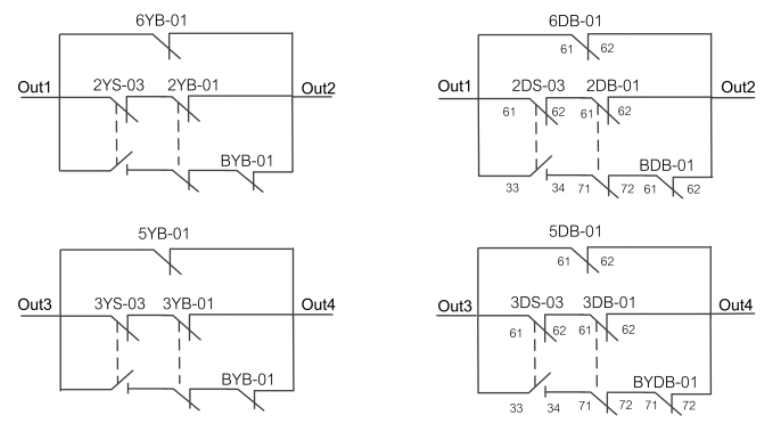

Trip circuit

(b)

Figure.10 Single diagram of the APB substation: (a) DTT function and (b) Trip matrix and Trip circuit

At OUT3 and OUT4 terminals, the trip signals will be sending out to open GCB 1YB-01 (LCA) when DS 3YS-03 (APB) or GCB 3YB-01 (APB) opened. Another case to send out the trip signals is when the transfer bus function is in use meaning that the GCB BYB-01 or GCB 5YB-01 are opened, see also Fig. 10 (b).

Fig. 11 represents the modified single line diagram, the logic algorithm of the trip matrix and trip circuit at LCA substation. This modification with the same concept as mentioned in Fig. 10. The details are as follows:

At OUT1 and OUT2 terminals, the trip signals will be sending out to open GCB 1YB-01 (LCA) when DS 1YC-03 (APB) or GCB 1YB-01 (APB) opened. Another case to send out the trip signals is when the transfer bus function is in use meaning that the GCB BYB-01 is opened; see also Fig. 11(b).

At OUT3 and OUT4 terminals, the trip signals will be sending out to open GCB 1YB-01 (LCA) when DS 2YS-03 (APB) or GCB 2YB-01 (APB) opened. Another case to send out the trip signals is when the transfer bus function is in use meaning that the GCB BYB-01 is opened; see also Fig. 11(b).

At OUT5 and OUT6 terminals, the trip signals 


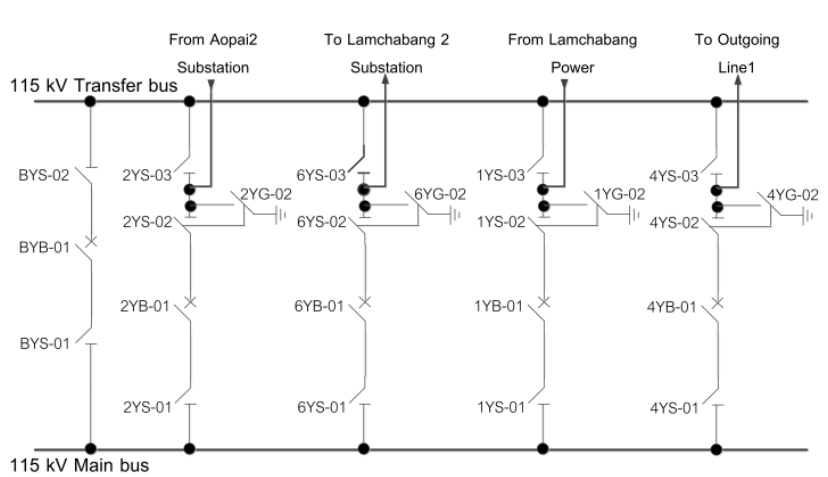

(a)
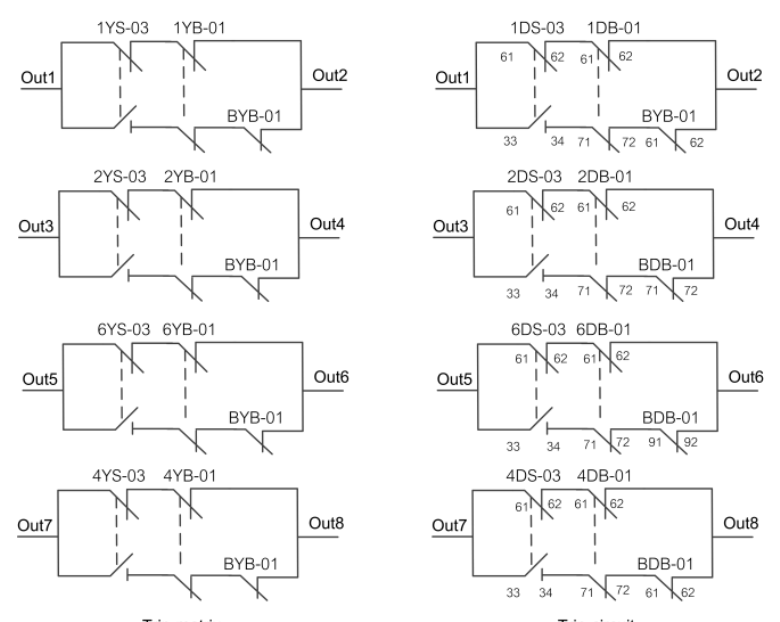

(b)

Figure.11 Single diagram of the LCA substation: (a) DTT function and (b) Trip matrix and trip circuit

will be sending out to open GCB 1YB-01 (LCA) when DS 6YS-03 (APB) or GCB 6YB-01 (APB) opened; see also Fig. 11(b).

Additional function at this substation, at Out7 and Out8, is to send out the trip signals to open GCB 1YB-01 (LCA) when DS 4YS-03 (LCA) or GCB 4YB-01 opened or when the transfer bus GCB BYB-01 is in functioned.

Fig. 12(a) represents the modified single line diagram, the logic algorithm of the trip matrix and trip circuit at LCB substation. The details of the trip matrix and trip circuits are as follows:

At OUT1 and OUT2 terminals, the trip signals will be sending out to open GCB 1YB-01 (LCA) when DS 5YS-03 (LCB) or GCB 5YB-01 (APB) opened. Another case to send out the trip signals is when the transfer bus function is in use meaning that the GCB BYB-01 is opened; see also Fig. 12(b).

At OUT3 and OUT4 terminals, the trip signals will be sending out to open GCB 1YB-01 (LCA) when DS 4YS-03 (LCB) or GCB 4YB-01 (APB) opened. Another case to send out the trip signals is

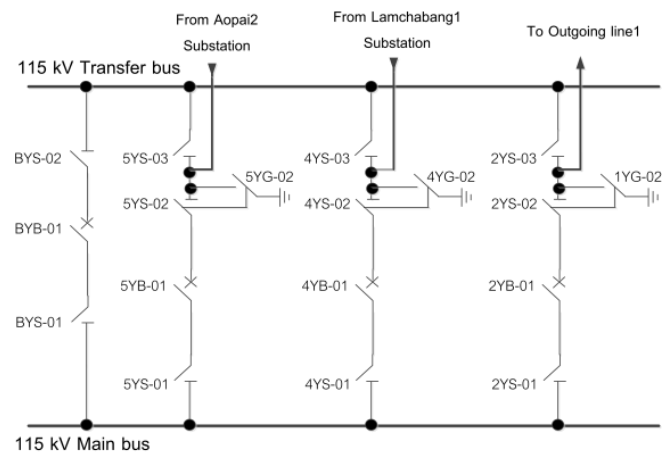

(a)
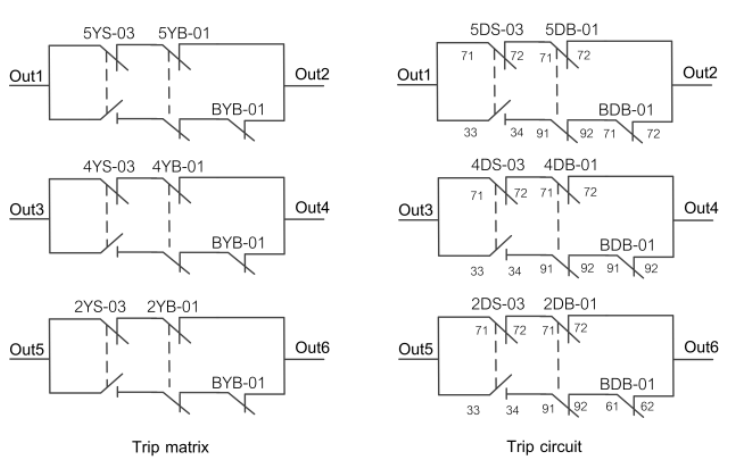

(b)

Figure.12 Single diagram of the LCB substation: (a) DTT function and (b) Trip matrix and trip circuit

when the transfer bus function is in use meaning that the GCB BYB-01 is opened; see also Fig. 12(b).

Additional function at this substation, at Out5 and Out6, is to send out the trip signals to open GCB 1YB-01 (LCA) when DS 2YS-03 (LCB) or GCB 2YB-01 opened or when the transfer bus GCB BYB-01 is in functioned.

\section{The proposed DTT test results}

In order to verify the proposed protection scheme validity and reliability, the commissioning test after installation is mandatory (this process is sometimes called FAT test). The commissioning test procedures utilize the hardware test tools (OMICRON CMC-356 universal relay test equipment) and also the special simulation software. The commissioning test was done under various fault conditions.

\subsection{DTT versus Peer-to-Peer logic test results}

Fig.13 illustrates system configuration for the FAT/commissioning test set up of teleprotection cabinets. This was done by simulating the connection between two teleprotection cabinets at both ends (APB and LCA) then connecting with actual devices in the simulation. The DTT with Mirrored bits ${ }^{\circledR}$ 


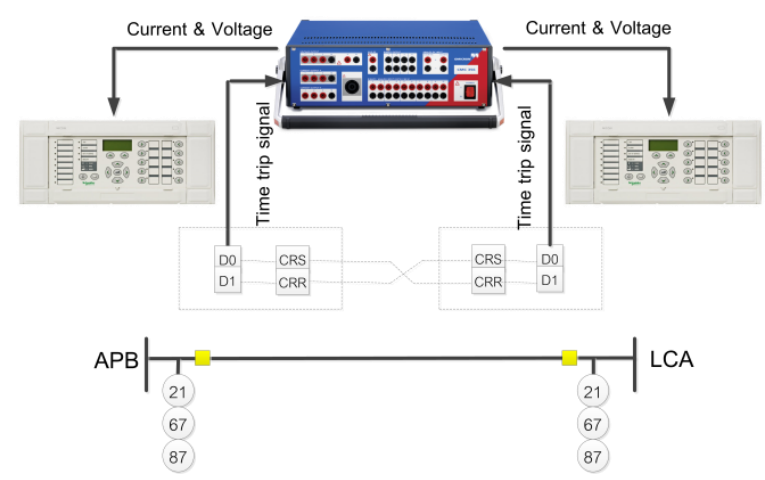

Figure.13 System configuration of RTU cabinet for the faults simulation and commissioning tests

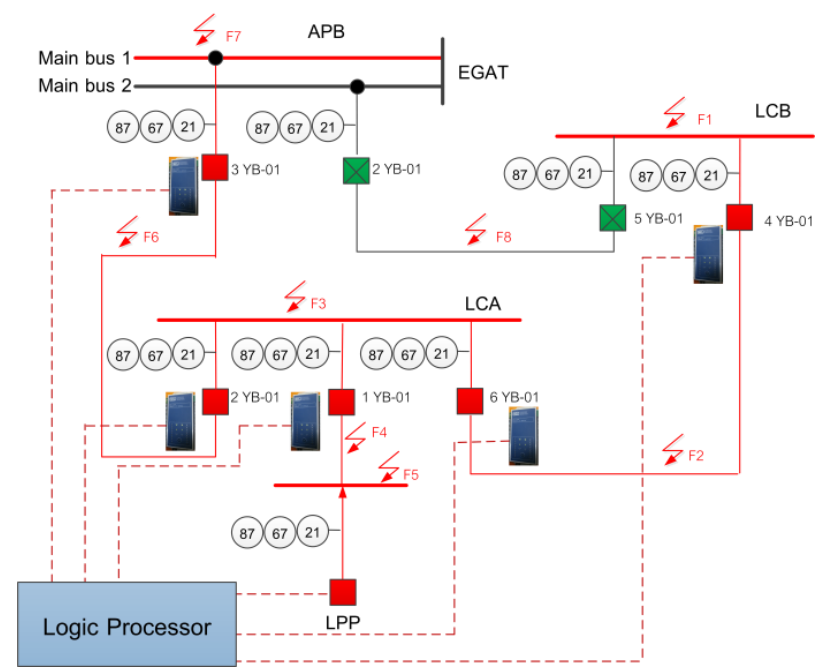

F1: Fault at LCB Bus

F2: Fault at Line between $L C A$ and $L C B$

F3: Fault at LCA Bus

F4: Fault at Line between $L C A$ and $L P$

F5: Fault at LP Bus

F6: Fault at Line between $L C A$ and $A P B$

F7: Fault at APB Bus

F8: Fault at Line between $A P B$ and $L C B$

Figure.14 Simulation fault locations for proposed DTT

protocol signals are applied to relay protections at each side. The injected voltage and current to the relay was created by a test tool (OMICRON CMC356). The DTT signal is also simulated by copper wires via Digital input/output (DI/DO) of teleprotection cabinets. The protective relay operating results will send out the signal to the logic processor via remote $\mathrm{I} / \mathrm{O}$ terminal. The operating time of all signals are measured by this procedure.

Fig. 14 presents a variety of possible fault locations at each of PEA closed-loop substations, Buses and Lines used in the commissioning test configurations.

The experimental results for the commissioning test of the DTT teleprotection functions compared to
Table 1. Commissioning test results for difference faults simulation with DTT protection scheme installation (115 kV incoming from Main Bus 1)

\begin{tabular}{|c|c|c|c|c|}
\hline Fault & $\begin{array}{c}\text { EGAT } \\
\text { Status }\end{array}$ & $\begin{array}{c}\text { Status mode } \\
\text { (LP) }\end{array}$ & $\begin{array}{c}\text { Peer- } \\
\text { to-Peer } \\
\text { (LP) }\end{array}$ & $\begin{array}{c}\text { DTT } \\
\text { (LP) }\end{array}$ \\
\hline F1 & In service & Normal & No trip & No trip \\
\hline F2 & In service & Normal & No trip & No trip \\
\hline F3 & In service & Island & No trip & Trip \\
\hline F4 & In service & Island & Trip & Trip \\
\hline F5 & In service & Island & Trip & Trip \\
\hline F6 & In service & Island & No trip & Trip \\
\hline F7 & $\begin{array}{c}\text { Out of } \\
\text { service }\end{array}$ & Island & No trip & Trip \\
\hline F8 & N/A & N/A & N/A & N/A \\
\hline
\end{tabular}

Table 2. Commissioning test results for difference faults simulation with DTT protection scheme installation (115 kV incoming from Main Bus 2)

\begin{tabular}{|c|c|c|c|c|}
\hline Fault & $\begin{array}{c}\text { EGAT } \\
\text { Status }\end{array}$ & $\begin{array}{c}\text { Status mode } \\
\text { (LP) }\end{array}$ & $\begin{array}{c}\text { Peer- } \\
\text { to-Peer } \\
\text { (LP) }\end{array}$ & $\begin{array}{c}\text { DTT } \\
\text { (LP) }\end{array}$ \\
\hline F1 & In service & Island & No trip & Trip \\
\hline F2 & In service & Island & No trip & Trip \\
\hline F3 & In service & Island & No trip & Trip \\
\hline F4 & In service & Island & Trip & Trip \\
\hline F5 & In service & Island & Trip & Trip \\
\hline F6 & N/A & N/A & N/A & N/A \\
\hline F7 & $\begin{array}{c}\text { Out of } \\
\text { service }\end{array}$ & Island & No trip & Trip \\
\hline F8 & In service & Island & No trip & Trip \\
\hline
\end{tabular}

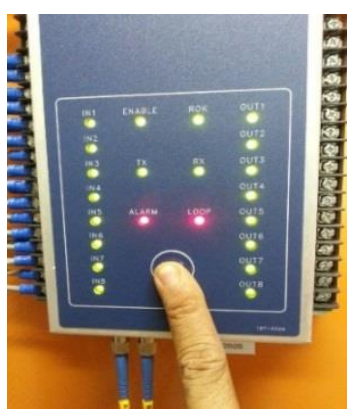

(a)

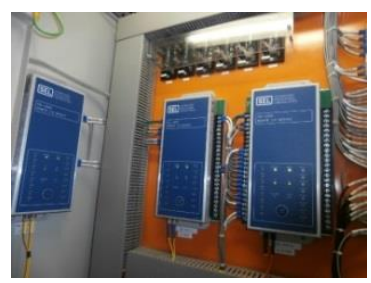

(c)

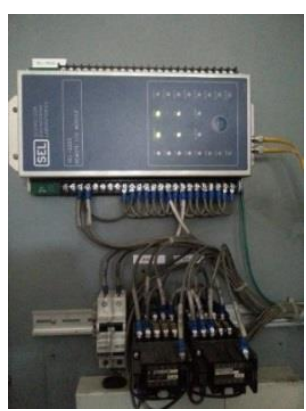

(b)

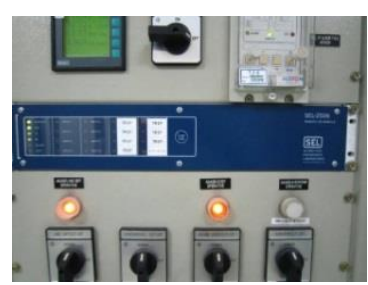

(d)
Figure.15 Actual system configuration of RTU cabinet and remote I/O units for the proposed DTT scheme: (a) Remote I/O unit (SEL-2505), (b) Remote I/O at LCA, (c) Remote I/O at LCA and (d) Logic processor at LPP 
the traditional and existing peer-to-peer protection scheme are shown in Table 1 and Table 2 and also can be seen in Fig. 15.

\subsection{DTT versus Peer-to-Peer tripping times and functions test results}

In this particular simulation test, the example of relay setting will be done only on distance relay. By dividing zone protection into four zones, distance relay protection settings at both APB and LPP terminals will be illustrated as follows:

- Zone 1: setting at $80 \%$ of transmission line with instantaneous trip time

- Zone 2: setting at 100\% + 20\% of transmission line with $300 \mathrm{~ms}$. trip time

- Zone 3: setting reverse with $300 \mathrm{~ms}$ trip time

- Zone 4: setting forward with $300 \mathrm{~ms}$ trip time

In this simulation test, fault currents and voltages (using relay tester) will be fed into distance relay protection in a particular zone at each substation: APB and LPP, Fig. 11. The simulation test results when injecting fault to each location can be seen from Table 3 (fault at APB) and Table 4 (Fault at LPP). The distance relay trip time of the

Table 3. Validation and trip time comparisons test results for distance relay when fault occurred at the power sources substation APB (at the PEA Line 1)

\begin{tabular}{|c|c|c|c|c|c|}
\hline \multirow{2}{*}{ Faults } & \multicolumn{3}{|c|}{$\begin{array}{c}\text { Traditional } \\
\text { Peer-to-Peer } \\
\text { (Only distance relay) }\end{array}$} & \multicolumn{2}{|c|}{$\begin{array}{c}\text { DTT } \\
\text { Technique } \\
\text { at LPP } \\
\end{array}$} \\
\hline & $\begin{array}{c}\text { Time } \\
\text { set } \\
(\mathrm{ms}) \\
\end{array}$ & $\begin{array}{c}\text { at APB } \\
(\mathrm{ms})\end{array}$ & $\begin{array}{c}\text { at } \\
\text { LPP } \\
(\mathbf{m s}) \\
\end{array}$ & $\begin{array}{c}\text { Time } \\
\text { set } \\
(\mathrm{ms}) \\
\end{array}$ & $\begin{array}{c}\text { Found } \\
(\mathrm{ms})\end{array}$ \\
\hline Zone 1 & Inst & 33.46 & \multirow{4}{*}{$\begin{array}{l}\text { Seen as } \\
\text { back } \\
\text { up } \\
\text { protect- } \\
\text { tion }\end{array}$} & Inst & 34.52 \\
\hline Zone 2 & 300 & 289.45 & & 300 & 299.84 \\
\hline Zone 3 & 300 & 299.35 & & 300 & 300.45 \\
\hline Zone 4 & 600 & 589.50 & & 600 & 600.62 \\
\hline
\end{tabular}

Table 4. Validation and trip time comparisons test results for distance relay when fault occurred at the LPP power plant

\begin{tabular}{|c|c|c|c|c|c|}
\hline \multirow{2}{*}{ Faults } & \multicolumn{3}{|c|}{$\begin{array}{c}\text { Traditional } \\
\text { Peer-to-Peer } \\
\text { (Only distance relay) }\end{array}$} & \multicolumn{2}{|c|}{$\begin{array}{c}\text { DTT } \\
\text { Technique } \\
\text { at LPP } \\
\end{array}$} \\
\hline & $\begin{array}{c}\text { Time } \\
\text { set } \\
(\mathrm{ms})\end{array}$ & $\begin{array}{c}\text { at APB } \\
(\mathrm{ms})\end{array}$ & $\begin{array}{c}\text { at } \\
\text { LPP } \\
(\mathbf{m s})\end{array}$ & $\begin{array}{l}\text { Time } \\
\text { set } \\
(\mathbf{m s})\end{array}$ & $\begin{array}{c}\text { Found } \\
(\mathrm{ms})\end{array}$ \\
\hline Zone 1 & Inst & \multirow{4}{*}{$\begin{array}{l}\text { Seen as } \\
\text { back up } \\
\text { protect- } \\
\text { tion }\end{array}$} & 37.21 & Inst & 38.05 \\
\hline Zone 2 & 300 & & 295.41 & 300 & 296.03 \\
\hline Zone 3 & 300 & & 285.68 & 300 & 286.32 \\
\hline Zone 4 & 600 & & 588.40 & 600 & 589.67 \\
\hline
\end{tabular}

conventional protection system will be directly measured at the fault location. The trip time of the DTT technique will be measured at the LPP only.

It can clearly be seen from the simulation test results shown in Table 3 and Table 4 that when there is any fault occurred out of Zone 1 protection, the conventional peer-to-peer protection scheme either detects the fault too slow or cannot detect the fault at all, resulting in Islanding mode operation of LPP's generators and may cause damages.

On the other hand, the proposed DTT technique with Mirrored Bits ${ }^{\circledR}$ protocol is able to sense any fault from remote location almost simultaneously even if when the fault is out of Zone 1 protection. Consequently, the GCB at LPP is able to open on time to prevent any damage from generators operating on Islanding mode. Also, the speed of operation with DTT protection scheme is excellent.

\section{Conclusions}

It can be clearly seen from the commissioning test results that the newly proposed DTT technique for this particular closed-loop distribution network works flawlessly. The proposed DTT technique completely covers in preventing generators at LPP from operating in islanding mode while the conventional peer-to-peer technique cannot in many fault circumstances. The simulation results also show that the proposed DTT time of operation is almost instantaneous regardless of fault locations. These result in better stability, speed and security of the distribution network. The same concept as presented in this paper would highly be beneficial for other similar power system protection with DGs in Thailand or other countries in the near future to come.

\section{ACKNOWLEDGMENTS}

The authors would like to thank Lamchabang Power, Thai power center Co., Ltd. and Mr. Athiruk Aungkum for kindly providing the partial and important information used in this research. The authors would also like to thank the ESIRC team members at KMITL for all of their support in many ways throughout this research. And lastly, the first author would like to give a special thanks to both of the Electrical engineering departments at KMITL and MUT for part of the financial supports.

\section{References}

[1] C. Chompoo-inwai, P. Fuangfoo, and W. Lee, "Transmission Congestion Management during Transition Period of Electricity Deregulation in 
Thailand", IEEE Transaction on Industrial Application, Vol.43, No.6, pp.1483-1490, 2007.

[2] D. Fischer and R. Madge, "Digital Teleprotection Units: A Technology Overview", IEEE Transactions on Power Delivery, Vol.7, No.4, pp.1769-1774, 1992.

[3] International Electrotechnical Commission, IEC 60834-1 Standard: Teleprotection Equipment of Power System Performance and Testing - Part 1: Command System, Ed. 2.0, 1999.

[4] International Electrotechnical Commission, IEC 60834-2 Standard: Performance and Testing of Teleprotection Equipment of Power Systems Part 2: Analogue Comparison Systems, 1993.

[5] E. Schweitzer, K. Behrendt, T. Lee, and D. A. Tziouvaras, "Digital Communications for Power System Protection: Security, Availability, and Speed", In: Proc. of $20017^{\text {th }}$ International Conference on Development in Power System Protection, Amsterdam, Netherland, pp.94-97, 2001.

[6] E. Schweitzer, D. Finney, and M. V. Mynam, "Applying Radio Communication in Distribution Generation Teleprotection Schemes", In: Proc. of $65^{\text {th }}$ Annual Conference for Protective Relay Engineers, College Station, TX, pp.310-320, 2012.

[7] J. Jager, M. Ramold, and S. Li, "Adaptive Protection Co-ordination Methods Concerning A Dedicated Operation of Large IPP Unit Connected to The Transmission Grid", In: Proc. of IEEE International Conference on Power System Technology, Changing, China, pp.1-4, 2006.

[8] A. Luiz and P. Oliveira, "The Use of Real Time Digital Simulation for Performance Analysis of Distance Protection and Differential Protection in Short Transmission Lines", In: Proc. of CIRED $20^{\text {th }}$ International Conference on Electricity Distribution, Prague, Czech \& Republic, pp.8-11, 2009.

[9] M. Khodadad and S. Shahrtash, "Guideline for Selecting Teleprotection Schemes for TOFFsCase Study: Iran Grid", In: IEEE 10 International Conference on Environment and Electrical Engineering, Rome, Italy, pp.1-5, 2011

[10] E. Schweitzer, D. Finney, and M. Mynam, "Applying Radio Communication in Distribution Generation Teleprotection Schemes", In: IEEE Annual Conference for Protective Relay Engineers, College Station, Texas, pp.310-320, 2012.

[11] G. Antonova, E. Colmenares, and I. Jankovic, "Analysis of Protection Scheme Dependencies on Communications", In: IEEE Annual Conference for Protective Relay Engineers, College Station, Texas, pp.271-291, 2013.

[12] S. Roesler and R. Lobo, "Proving Viability of Line Current Differential over Packing Switched Networks", In: IEEE $67^{\text {th }}$ Annual Conference for Protective Relay Engineers, College Station, Texas, pp.542-551, 2014.

[13] C. Chompoo-inwai, A. Aungkum, N. Chotiwanaporn, and M. Leelajindakrairerk, "A New Distributed Generation Protection Scheme in Thailand Using Direct Transfer Trip (DTT) Technique and A Mirrored Bit Protocol", Journal of International Council on Electrical Engineering, Vol.5, No.1, pp.34-41, 2015. 